\title{
The Relationship Between Oseltamivir and Suicide in Pediatric Patients
}

Rachel Harrington, $B A^{1}$

Srutbi Adimadhyam, $M S^{1}$

Todd A. Lee, PbarmD, PbD ${ }^{1}$

Glen T. Schumock, PharmD, MBA, $P h D^{1}$

James $W$. Antoon, $M D, P b D^{2}$

'University of Illinois at Chicago College of Pharmacy, Chicago, Illinois

${ }^{2}$ Children's Hospital University of Illinois, Chicago, Illinois
Conflicts of interest: authors report none.

\section{CORRESPONDING AUTHOR}

James W. Antoon, MD, PhD 840 S. Wood St M/C 856

Chicago, IL 60612

jantoon@uic.edu

\begin{abstract}
PURPOSE Studies examining the association between use of oseltamivir and neuropsychiatric events (including suicide) among children have had mixed findings and have been limited by small sample size, reliance on older data, and potential confounding. We undertook an analysis that addresses these limitations.
\end{abstract}

METHODS Using a national administrative claims database and a case-crossover design that minimized confounding, we analyzed data from 5 contemporary influenza seasons (2009-2013) for individuals aged 1 to 18 years and ascertained oseltamivir exposure from pharmacy dispensing.

RESULTS We identified 21,407 suicide-related events during this study period, 251 of which were in oseltamivir-exposed children. In case-crossover analysis, we did not find any significant association with suicide either for oseltamivir exposure (odds ratio $=0.64 ; 95 \% \mathrm{Cl}, 0.39-1.00 ; P=.05$ ) or for influenza diagnosis alone (odds ratio $=0.63 ; 95 \% \mathrm{Cl}, 0.34-1.08 ; P=.10$ ).

CONCLUSION Our findings suggest that oseltamivir does not increase risk of suicide in the pediatric population.

Ann Fam Med 2018;16:145-148. https://doi.org/10.1370/afm.2183.

\section{INTRODUCTION}

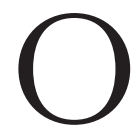
seltamivir is an antiviral agent used to treat influenza types A and B. Originally approved in 1999, it is routinely prescribed in pediatric practice. About 4 in 10 oseltamivir prescriptions dispensed in the United States between 2005 and 2011 were to children aged 16 years or younger. ${ }^{1}$

Case reports from the early 2000s raised concerns regarding the potential for psychiatric adverse events in children being given oseltamivir. Reported events included abnormal behavior, psychosis, and suicide, resulting in a change to the package insert in 2006 to include the risk of these events. ${ }^{2}$ Published evidence of this association is not consistent, however. Secondary analyses of randomized controlled trials among pediatric patients and some observational cohort studies have not found an association. ${ }^{3-5}$ Case reports, observational studies, and analyses of the Food and Drug Administration's Adverse Event Reporting System do suggest a potential risk, however. ${ }^{6-8}$

Prior US observational studies of a link between oseltamivir and suicide have examined older data (through the early 2000s) or a single influenza season, and have included suicide only as a secondary outcome. ${ }^{4,5}$ Moreover, these studies' designs were unable to account for important clinical and sociodemographic variables, suggesting that findings may have been influenced by unmeasured confounding.

The main objective of this study was to assess whether there is an association between oseltamivir and suicide attempt in pediatric patients using data from more recent influenza seasons and a novel study design to address confounding concerns. We also assessed influenza alone as a possible confounding risk factor for pediatric suicide. 


\section{METHODS}

We used a case-crossover design, whereby individual cases serve as their own control, to minimize confounding. ${ }^{9}$ Five influenza seasons (2009-2013), defined as October 1 of one year through April 30 of the subsequent year, were examined.

The study was conducted using the Truven MarketScan Commercial Claims and Encounters database, an administrative claims database consisting of 50 million beneficiaries across all 50 states. ${ }^{10}$ The University of Illinois Institutional Review Board determined that the study did not met the definition of human subjects research, and it was therefore exempted.

Inclusion criteria were an age of 1 to 18 years at the time of the suicide-related event (index), 6 months of prior continuous enrollment, and an inpatient or outpatient suicide diagnosis during the influenza season. Children were allowed to contribute to multiple influenza seasons. A suicide-related event was defined as presence of a first suicide-related International Classification of Diseases, Ninth Revision, Clinical Modification (ICD9-CM) code of $\mathrm{E}$, denoting external cause of injury, or a code of V62.84, Suicidal Ideation. ${ }^{4}$

We ascertained oseltamivir exposure from outpatient pharmacy dispensing and exposure duration from the number of days supplied that was listed in the claim. For each patient, we assigned the 10-day period immediately before the index event as the case period, and we identified up to 4 earlier control periods of the same length (Figure 1). As in case-control studies that match a single case to many controls, we used multiple control periods to improve precision (eg, obtain tighter CIs) and our ability to detect a potential effect. ${ }^{11}$ Control periods were selected from the same influenza season as the case period, with the number of control periods determined by the time available between start of the season and the event date. Exposure status was determined in both case and control periods. Durations of the case and control periods were selected based on typical oseltamivir dosing and half-life. In sensitivity analyses, the exposure and control periods were reduced (5-day exposure and control periods, 10 days of washout), and expanded (15-day exposure and control periods, 15 days of washout). We conducted an additional sensitivity analysis restricting the eligible event period to January 1 through March 31 of each influenza season to examine the effect of case-event timing during the season.

An observed association between oseltamivir and suicide could potentially be confounded by underlying influenza infection. To evaluate such potential confounding by indication, we performed a secondary analysis using influenza diagnosis alone (without oseltamivir use) as the exposure. The duration of influenza exposure was set at 7 days.

We calculated odds ratios (ORs) and 95\% confidence intervals for the effects of oseltamivir (vs no oseltamivir) and influenza (vs no influenza) using conditional logistic regression analysis. Because of the case-based design, it was not necessary to adjust for covariates in this analysis. Results were stratified by hypothesized effect modifiers, measured over the 6 -month baseline period before the event. Data management and analyses were conducted using SAS 9.4 (SAS Institute).

\section{RESULTS}

We identified 21,047 individuals aged 1 to 18 years who attempted suicide during the study period and qualified for the analysis; of those, 251 were exposed to oseltamivir at the time. The mean age of this exposed

\section{Figure 1. Study design schematic.}

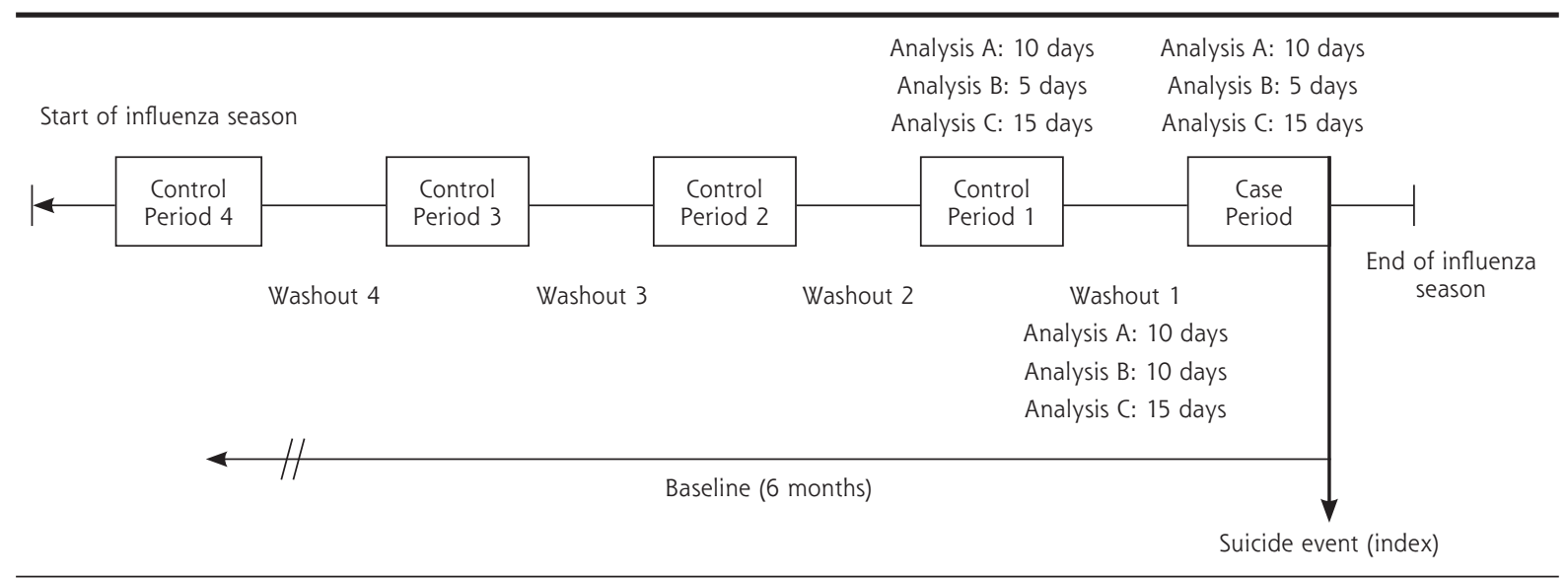

Note: For a given analysis, all control time periods are equal to case periods in length, and all washout periods are the same duration. 
group was approximately 15 years, and $61 \%$ were female. Underlying mental health diagnoses were common (65\%).

In the primary analysis, the odds ratio of oseltamivir exposure during the case period before the event was 0.64 (95\% CI, 0.39-1.00; $P=.05$ ) (Table 1).

In the secondary analysis, we identified 162 individuals aged 1 to 18 years exposed only to influenza. Their characteristics were similar to those of the oseltamivir-exposed group. The odds ratio for suicide attempt for influenzaonly exposure, vs no influenza, during the case period was $0.63(95 \% \mathrm{CI}$, 0.34-1.08; $P=.10)$.

Effect estimates from sensitivity analyses that examined alternative case and control time periods were similar in magnitude and direction to those of the primary analysis. Two findings were statistically significant: a 15-day window analysis of oseltamivir exposure $(\mathrm{OR}=0.57$; 95\% CI, 0.36-0.86; $P=.007)$ and a 5 -day window analysis of influenza-only exposure $(\mathrm{OR}=0.48$; CI, 0.22-0.95; $P=.03)$. Estimates from analysis restricting the eligible event period to January 1 through March 31 of each influenza season were also in the same direction as those of the primary analysis.

\section{DISCUSSION}

We did not find a significantly increased risk between suicide-related events and the use of oseltamivir. This result is consistent with those from previous studies that used different study designs, and contrasts with the warning in the package insert. ${ }^{2,4,5}$ The association for influenza-only exposure was of similar direction and magnitude as that for oseltamivir exposure, suggesting no confounding by indication of underlying influenza.

The findings of our study did not change in the sensitivity analyses.

Although we found a statistically significant reduction in risk with some alternative case and control time periods, results should be interpreted with caution. For instance, the 15-day window analysis of oseltamivir, although biologically plausible, extends the drug's

\section{Table 1. Association Between Exposure to Oseltamivir or Influenza Alone and Suicide: Overall and Stratified by Individual Characteristics}

\begin{tabular}{|c|c|c|}
\hline Characteristic & Oseltamivir & Influenza Only \\
\hline \multicolumn{3}{|c|}{ Analysis A: 10 -day equivalent periods } \\
\hline Case, No. & $253^{\mathrm{a}}$ & $173^{a}$ \\
\hline Exposed & 24 & 17 \\
\hline Unexposed & 229 & 156 \\
\hline Control, No. & 949 & 647 \\
\hline Exposed & 124 & 89 \\
\hline Unexposed & 825 & 558 \\
\hline Overall, OR (95\% Cl) & $0.64(0.39-1.00)^{b}$ & $0.63(0.34-1.08)$ \\
\hline \multicolumn{3}{|l|}{ Sex, OR $(95 \% \mathrm{Cl})$} \\
\hline Male & $0.64(0.31-1.32)$ & $0.82(0.36-1.86)$ \\
\hline Female & $0.64(0.34-1.05)$ & $0.52(0.25-1.07)$ \\
\hline \multicolumn{3}{|l|}{ Age-group, OR (95\% Cl) } \\
\hline $1-11$ y & $0.50(0.11-2.27)$ & $0.40(0.04-3.69)$ \\
\hline $12-18$ y & $0.66(0.41-1.04)$ & $0.65(0.37-1.12)$ \\
\hline \multicolumn{3}{|l|}{ Mental health history, OR (95\% CI) } \\
\hline No & $0.62(0.35-1.08)$ & $0.51(0.26-1.00)$ \\
\hline Yes & $0.68(0.33-1.42)$ & $0.99(0.39-2.45)$ \\
\hline \multicolumn{3}{|c|}{ History of suicide attempt, OR $(95 \% \mathrm{Cl})$} \\
\hline No & $0.62(0.39-0.99)$ & $0.30(0.04-2.38)$ \\
\hline Yes & $0.91(0.18-4.53)$ & $0.67(0.39-1.18)$ \\
\hline \multicolumn{3}{|c|}{ Influenza vaccine during season, OR (95\% Cl) } \\
\hline No & $0.71(0.45-1.13)$ & $0.60(0.33-1.07)$ \\
\hline Yes & $0.19(0.03-1.46)$ & $0.84(0.22-3.19)$ \\
\hline \multicolumn{3}{|c|}{$\begin{array}{l}\text { Analysis B: } 5 \text {-day case period, 10-day } \\
\text { washout, 5-day control period }\end{array}$} \\
\hline Case, No. & 253 & 173 \\
\hline Exposed & 16 & 10 \\
\hline Unexposed & 237 & 163 \\
\hline Control, No. & 985 & 673 \\
\hline Exposed & 74 & 74 \\
\hline Unexposed & 911 & 599 \\
\hline Overall association, OR (95\% Cl) & $0.82(0.45-1.43)$ & $0.48(0.22-0.95)^{c}$ \\
\hline \multicolumn{3}{|c|}{ Analysis C: 15 -day equivalent periods } \\
\hline Case, No. & 253 & 173 \\
\hline Exposed & 28 & 24 \\
\hline Unexposed & 225 & 149 \\
\hline Control, No. & 886 & 595 \\
\hline Exposed & 150 & 96 \\
\hline Unexposed & 736 & 499 \\
\hline Overall association, OR (95\% Cl) & $0.57(0.36-0.86)^{d}$ & $0.72(0.43-1.15)$ \\
\hline \multicolumn{3}{|l|}{$\mathrm{OR}=$ odds ratio. } \\
\hline \multicolumn{3}{|c|}{$\begin{array}{l}\text { a Individuals were allowed to contribute multiple cases, as long as they occurred in different influenza } \\
\text { seasons. The } 253 \text { oseltamivir cases were contributed by } 251 \text { individuals. The } 173 \text { influenza cases were } \\
\text { contributed } 162 \text { individuals. } \\
\text { b } P=.05 \text {. } \\
\text { c } P<.05 \text {. } \\
\text { d } P<.01 .\end{array}$} \\
\hline
\end{tabular}

effect duration to its extreme. This extension may lead to an exaggeration of the effect due to misclassification of the case period as "exposed."

By leveraging the case-crossover design, we were able to account for within-person confounders (eg, 
trauma, abuse, and baseline psychiatric status) and time-invariant confounders (eg, race), irrespective of their availability in the data. This design addresses drawbacks of previous studies examining neuropsychiatric effects of oseltamivir.

Our results are subject to a number of limitations, however. Suicide-related events may be underidentified, as claims data capture only events that result in a billed medical encounter. Patients with recorded events may be less likely to use health care in the days immediately preceding the event, potentially resulting in the observed null effect. Although case reports suggest a possible higher oseltamivir-related risk in the Asian population, we were unable to examine effect modification by race because the data set does not capture this characteristic. Our decision to define the influenza season according to the same dates across years, while unlikely to introduce bias into the study results, does not reflect the true variability in season duration and may have artificially restricted our sample size. Finally, although we made multiple comparisons, we did not make corrections for multiple testing. As the main study finding was the lack of significant effect, we do not believe the use of a more conservative alpha would change the interpretation of study results. This possibility highlights the importance of considering effect size and CIs, however, when assessing potential harms.

Despite the above limitations, our results suggest that oseltamivir does not increase the risk of suicide in the pediatric population.

To read or post commentaries in response to this article, see it online at http://www.AnnFamMed.org/content/16/2/145.

Key words: oseltamivir; suicide; self-harm; pediatric; observational; influenza; adverse effects

Submitted June 29, 2017; submitted, revised, September 20, 2017; accepted October 4, 2017.
Funding support: Rachel Harrington was supported by $\mathrm{NCl}$ training grant 5R25-CA057699. The Department of Pharmacy Systems, Outcomes and Policy and the Center for Pharmacoepidemiology and Pharmacoeconomic Research at the University of Illinois at Chicago, Chicago, Illinois, provided access to the Truven Health Marketscan Commercial Database used in this study.

\section{References}

1. Taylor A. Tamiflu (oseltamivir phosphate) Background Information and Drug Utilization Patterns. https://wayback.archive-it.org/7993/ 20170114054320/http://www.fda.gov/downloads/AdvisoryCommittees/ CommitteesMeetingMaterials/PediatricAdvisoryCommittee/ UCM303004.pdf. Published May 2012. Accessed Feb 22, 2017.

2. Tamiflu [package insert]. South San Francisco, CA: Genentech, Inc; 2016. https://dailymed.nlm.nih.gov/dailymed/druglnfo.cfm? setid $=$ ee3c9555-60f2-4f82-a760-11983c86e97b. Published Jun 30, 2016. Accessed Feb 24, 2017.

3. Jefferson T, Jones MA, Doshi $P$, et al. Neuraminidase inhibitors for preventing and treating influenza in adults and children. Cochrane Database Syst Rev. 2014;(4):CD008965. http://onlinelibrary.wiley. com/doi/10.1002/14651858.CD008965. pub4/abstract. Accessed Feb 13, 2017.

4. Blumentals WA, Song $X$. The safety of oseltamivir in patients with influenza: analysis of healthcare claims data from six influenza seasons. MedGenMed. 2007;9(4):23.

5. Casscells SW, Granger E, Kress AM, Linton A. The association between oseltamivir use and adverse neuropsychiatric outcomes among TRICARE beneficiaries, ages 1 through 21 years diagnosed with influenza. Int J Adolesc Med Health. 2009;21(1):79-89.

6. Hoffman KB, Demakas A, Erdman CB, Dimbil M, Doraiswamy PM. Neuropsychiatric adverse effects of oseltamivir in the FDA Adverse Event Reporting System, 1999-2012. BMJ. 2013;347(jul23 3):f4656.

7. Yorifuji T, Suzuki E, Tsuda T. Oseltamivir and abnormal behaviors: true or not? Epidemiology. 2009;20(4):619-621.

8. Nakamura Y, Sugawara T, Ohkusa Y, et al. Life-threatening abnormal behavior incidence in 10-19 year old patients administered neuraminidase inhibitors. PLoS One. 2015;10(7):e0129712.

9. Delaney JA, Suissa S. The case-crossover study design in pharmacoepidemiology. Stat Methods Med Res. 2009;18(1):53-65.

10. Hansen LG, Chang S. Health Research Data for the Real World: The MarketScan Databases. Ann Arbor, MI: Truven Health Analytics. http:// truvenhealth.com/Portals/O/Assets/PH_11238_0612_TEMP_Market Scan_WP_FINAL.pdf. Published Jul 2011. Accessed May 10, 2017.

11. Maclure M, Mittleman MA. Should we use a case-crossover design? Annu Rev Public Health. 2000;21(1):193-221. 